\title{
Restless legs syndrome and functional limitations among American elders in the Health and Retirement Study
}

\author{
Dominic J Cirillo ${ }^{1,2^{*}+}$ and Robert B Wallace ${ }^{1,2+}$
}

\begin{abstract}
Background: Restless legs syndrome (RLS) is a common condition associated with decreased quality of life in older adults. This study estimates the prevalence, risk factors, and functional correlates of among U.S. elders.

Methods: Subjects $(n=1,008)$ were sub-sampled from the 2002 cross-sectional interview survey of the Health and Retirement Study (HRS), a nationally representative study of U.S. elders. Symptoms and sleep disturbances consistent with RLS were identified. Activities of daily living (ADL), instrumental activities of daily living (IADL), and limitations for mobility, large muscle groups, gross and fine motor function were measured using standardized questions. Incident functional limitations were detected over six years of observation.

Results: The prevalence of RLS among U.S. elders born before 1947 was 10.6\%. Factors associated with increased prevalence RLS at baseline included: overweight body mass index (multivariate adjusted prevalence ratio $=1.77$; 95\% confidence interval (Cl) $1.05-2.99)$; mild-to-moderate pain $(2.67,1.47-4.84)$ or pain inferring with activity $(3.44,2.00-5.93)$; three or more chronic medications $(2.54,1.26-5.12)$, highest quartile of out-of-pocket medical expenses $(2.12,1.17-3.86)$, frequent falls $(2.63,1.49-4.66)$, health limiting ability to work $(2.91,1.75-4.85)$, or problems with early waking or frequent wakening $(1.69,1.09-2.62$ and 1.55, 1.00-2.41, respectively). Current alcohol consumption $(0.59,0.37-0.92)$ and frequent healthcare provider visits $(0.49,0.27-0.90)$ were associated with decreased RLS prevalence. RLS did not predict incident disability for aggregate measures but was associated with increased risk for specific limitations, including: difficulty climbing several stair flights (multivariate-adjusted hazard ratio $=2.38,95 \% \mathrm{Cl} 1.39-4.06)$, prolonged sitting $(2.17,1.25-3.75)$, rising from a chair $(2.54,1.62-3.99)$, stooping $(2.66,1.71-4.15)$, moving heavy objects $(1.79,1.08-2.99)$, carrying ten pounds $(1.61,1.05-2.97)$, raising arms $(1.76,1.05-2.97)$, or picking up a dime $(1.97,1.12-3.46)$.
\end{abstract}

Conclusions: RLS sufferers are more likely to have functional disability, even after adjusting for health status and pain syndrome correlates.

\section{Background}

Restless Legs Syndrome (RLS) is characterized by unpleasant sensations of tingling, creeping, bubbling, or "tunneling bugs" [1]. The International RLS Study Group established diagnostic criteria for RLS, including: a strong urge to move, predominantly in the legs; relief with movement, stretching, or rubbing; and symptomatic

\footnotetext{
* Correspondence: dominic-cirillo@uiowa.edu

${ }^{\dagger}$ Equal contributors

'Department of Internal Medicine, University of lowa Carver College of Medicine, lowa City, IA, USA

${ }^{2}$ Department of Epidemiology, University of lowa College of Public Health, lowa City, IA, USA
}

(c) 2012 Cirillo and Wallace; licensee BioMed Central Ltd. This is an Open Access article distributed under the terms of the Creative Commons Attribution License (http://creativecommons.org/licenses/by/2.0), which permits unrestricted use, distribution, and reproduction in any medium, provided the original work is properly cited. worsening at night or with inactivity [2]. RLS is a common chronic condition, with prevalence estimates ranging from $5-10 \%[3,4]$, with some geographic variability [3,5-7]. Many RLS cases are secondary to other causes, such as iron deficiency, pregnancy, and neurologic, rheumatologic, or renal diseases, but more than $60 \%$ of cases are idiopathic $[8,9]$. Primary RLS prevalence in the U.S. is likely $1-3 \%$ [9], and is likely more common in women and during the seventh and eighth decades [5]. RLS is clinically diagnosed but can be confirmed by polysomnography or the Suggested Immobilization Test [8], and no confirmatory laboratory tests exist [10]. Many RLS sufferers seek care but the average time to diagnosis is two 
years [11], and the RLS Epidemiology, Symptoms, and Treatment (REST) study confirmed that many are misdiagnosed and given inappropriate treatments [3].

RLS also impacts health-related quality of life [12-14]. Like those with other chronic conditions, RLS sufferers have lower scores on the Medical Outcome Study Short Form 36 (SF-36) in both clinical [15] and populationbased samples [5,12]. RLS sufferers may be less productive at work [9]. RLS sufferers report negative influences on mood, energy, and daily activities [5], and are shown to have more daytime fatigue, work difficulties, and driving impairment [4]. Among elders, severe RLS has been associated with poorer social function, daily function, sleep quality, and emotional well-being [16]. However, the full impact RLS has on disability has yet to be shown.

The Health and Retirement Study (HRS) is a nationally representative cohort of economic and physical health of elders [17]. Since 1992, HRS has provided comprehensive and detailed information on many domains, including health status, employment, disability, and net worth of U.S. elders. A sub-sample was queried about RLS symptoms in 2002, providing an opportunity to report epidemiologic correlates of RLS and, more importantly, longitudinal associations between RLS and subsequent functional limitations.

\section{Methods}

The Health and Retirement Study is an ongoing cohort study consisting of a representative sample of over 20,000 Americans born prior to 1948, with interview data collected biennially on demographics, health status, employment, income and wealth, and insurance; full details are described elsewhere [17]. In 2002, the overall response rate of the cohort was $86.9 \%$ [17]. Sub-samples ("modules") are asked additional questions on ancillary topics after the main survey [18]. The module topic is revealed after respondents consent. Of 1,502 randomly selected respondents, 1,058 (70.4\%) agreed to complete the 2002 RLS module. Nursing home residents and subjects outside the eligible age-range for HRS (e.g. spouses of age-eligible respondents) were excluded from the analysis $(n=50)$, yielding a final sample size of 1,008 . HRS was reviewed by the University of Michigan Institutional Review Board [17].

The 2002 RLS module predated validated instruments for measuring RLS outside of a clinical setting [2]. Respondents reported presence of resting symptoms, including "crawling, tingling or achy sensations in your arms or legs", feeling "restless, fidgety, or unable to sit still", "feel the need to move your legs, rub your legs, or stretch your legs", "you or bedpartner noticed twitching or kicking of your arms and legs", or "itching sensations anywhere on your body." Respondents reported how frequently they experienced "unpleasant feelings" in the legs - "for example, creepy-crawling or tingly feelingsthat make you feel restless and keep you from getting a good night's sleep", with options ranging from "never" to "every night". Subjects were also asked how frequently they "get pain or cramps in [the] legs to the point where it is uncomfortable and disturbs sleep". Symptomatic individuals were asked if they sought medical advice, whether treatment was offered, and whether treatment helped.

Potential health correlates were derived from the parent HRS study, and full questionnaires are available at http://hrsonline.isr.umich.edu [17]. Subjects self-reported demographic information, including race, gender, date of birth, and educational attainment, upon study entry. Health status is evaluated with each interview wave, including medication use, chronic medical conditions (hypertension, diabetes, heart disease, stroke, lung disease, psychiatric disease, arthritis, and cancer), health behaviors, and symptoms, including general sleep concerns and pain. Bodily pain experience was categorized into three levels: severe (pain makes normal activities difficult), mild-moderate (troubled by pain but does not limit activity), or no troubling pain. Pain site was not localized. General sleep-related concerns include difficulty falling asleep, waking frequently at night, and waking early and unable to return to sleep. Depressive symptoms were assessed using a shortened version of the Center for Epidemiologic Studies Depression (CES-D) Scale [19], with depressive symptoms noted for scores of two or more (corresponding to the $20^{\text {th }}$ most depressed percentile).

Smoking status was current, former, or never. Alcohol use was classified as current (yes/no), with additional quantification of drinking days per week and drinks per drinking day. Body mass index (BMI) was calculated using reported weight and height, and categorized as $\mathrm{BMI}<25,25 \leq \mathrm{BMI}<30$, or $\mathrm{BMI} \geq 30$ (18 missing values were classified at the median, 26.6). Self-reported health status was classified as "Excellent", "Very Good", "Good", "Fair", or "Poor". For this analysis, the top two and the bottom two response categories were collapsed. Selfreported vigorous activity at baseline was defined as at least three vigorous activity sessions per week. In subsequent waves, frequency of physical activity was further broken into episodes of mild, moderate, or vigorous activity per week. Individuals reported paid employment outside the home and whether personal health limited their ability to work. Income was calculated from all sources, and divided into quartiles. Total medical expenses and out-of-pocket medication expenses were collected, imputed as necessary from the RAND datafiles, and divided into quartiles. Subjects self-reported falls, hospitalizations, and frequency of healthcare provider 
visits in the previous two years. For the analysis, falls were grouped as "None", "One", or "Two or more". Frequency of provider visits were grouped as "0-3", "4-7", "8-11", or "12 or more". Self-reported medications for each of the above chronic conditions were queried, and for this analysis were categorized as "None", "One", "Two", or "Three or more".

Functional status was assessed using several standardized instruments. Variables were dichotomized to "at least some difficulty" versus no difficulty. Activities of daily living (ADL) assessed any difficulties on five tasks: bathing, eating, dressing, toileting, and transferring. Instrumental activities of daily living (IADL) included difficulties with: managing money, managing medications, preparing meals, going shopping, or using a telephone. Mobility limitations included limitations on walking and climbing stairs. Additional motor limitations were grouped based on a priori definitions. Gross motor limitations included any difficulty walking one block, climbing one flight of stairs, transferring, bathing, or requiring a walking assist device. Fine motor limitations included difficulty picking up a dime, feeding oneself, or dressing. Large muscle group limitations included sitting for two hours, rising from chair, stooping, or pushing a heavy object.

\section{Statistical analysis}

Analyses were performed using SAS, version 9.2 (SAS Institute, Inc., Cary, NC). RAND HRS analytic files, which provided processed data and derived variables [17], were linked to raw questionnaire data. The operational definition of RLS required all three criteria: a) feeling restless, fidgety, or unable to sit still; b) the need to move your legs, rub your legs, or stretch your legs; and c) unpleasant nighttime sensations occurring at least once per week. Crude cross-sectional RLS associations with categorical demographic variables were tested using $x^{2}$. Ordinal variables were evaluated for trend. Prevalence estimates were weighted for study sampling to reflect the U.S. non-institutionalized population born before 1947, reflecting the underlying HRS cohort.

Potential RLS correlates at baseline were explored by calculating multivariate-adjusted prevalence ratios (PR) derived from Cox proportional hazards regression with robust variance estimates [20]. Dependent variables were modeled with a fixed time effect (uniformly set to 1 ). Categorical predictors were screened and considered potentially important if the likelihood ratio test was significant at $\mathrm{p}<0.20$, using forward variable selection and comparisons of the Akaike Information Criteria (AIC). Continuous independent variables were tested in various manners (linear, polynomial, ordinal, or nominal categorical), with selection of best fitting relationship based on AIC. Correlation among predictors was evaluated based on eigenvalues derived from principal component analysis of the predictor variables. Variables were selected so that that each variable contributed uniquely to total variance (i.e., condition indices associated with individual predictor variables were $\leq 30$ ), while maximizing AIC [21]. Additional model-building approaches, such as chunkwise variable selection [22], did not significantly alter the final model.

RLS was then considered as an independent variable, adjusting for significant correlates from the RLS prediction model, to predict concurrent functional limitations. Disability variables were modeled as above to calculate multivariate-adjusted PR. Multi-domain measures (e.g., ADL, IADL) were positive if any component showed limitations. Next, using longitudinal disability data from three subsequent interview waves $(2004,2006$, and 2008), RLS was modeled as a predictor of incident functional limitations using Cox proportional hazards regression. Hazard ratios (HR) were reported for each incident disability, limited to the sample at risk (i.e., those without the specific functional limitation in 2002). Censoring occurred at the end of follow-up, death, voluntary withdrawal from the study, or first occurrence study ineligibility (i.e., living in an institutional setting or no longer being married to a spouse in the study cohort). Adjustment for pain and medication use at baseline had the greatest effect on the point estimates. Repeated measures were considered time-varying covariates in the analysis.

\section{Results}

Table 1 reports the baseline characteristics of the sample, with crude tests association. Symptoms of RLS were common (Table 2). The overall prevalence of restless legs syndrome using the operational case definition was $10.6 \%$. RLS was more prevalent in women $(\mathrm{p}<0.001)$, and individual symptoms were more common in women $(p<0.05)$, with the exception of "twitching or kicking", which was more frequent in men $(\mathrm{p}<0.05)$. RLS prevalence decreased with increasing age (test for trend, $\mathrm{p}<0.05$ ). There were no associations with race (data not shown). Among those with any symptoms, $43.4 \%$ discussed their symptoms with a doctor. Of these individuals, $40.4 \%$ reported receiving treatment for their symptoms. Women were significantly more likely than men to receive treatment. $78.8 \%$ of those who received treatment felt it was successful. Of the treatments mentioned in open-ended questioning (allowing for multiple responses), prescription medication was the most common (26\%), followed by exercise / physical therapy / rest (15\%), over-the-counter pain medications such as aspirin or ibuprofen (13\%), surgery / epidural (7\%), topical ointments, heat, or ice (5\%), support stockings / ankle weights $(4 \%)$, vitamins or minerals (4\%), change or 
Table 1 Characteristics of 2002 Health and Retirement Study restless legs syndrome module $(\mathrm{N}=1,008)$

\begin{tabular}{|c|c|c|c|c|c|c|}
\hline Variable & Included $(n=1008)$ & $\left(\%{ }^{*}\right)$ & $\operatorname{RLS}(n=104)$ & $\left(\%{ }^{*}\right)$ & No RLS $(n=904)$ & $\left(\%^{*}\right)$ \\
\hline \multicolumn{7}{|l|}{ Age } \\
\hline Age $54-64$ y & 374 & 46.6 & 43 & 52.5 & 331 & 45.9 \\
\hline Age $65-79$ y & 482 & 40.2 & 49 & 37.0 & 433 & 40.6 \\
\hline Age 80 y or older & 152 & 13.2 & 12 & 10.5 & 140 & 13.5 \\
\hline \multicolumn{7}{|l|}{ Gender : } \\
\hline Male & 430 & 44.4 & 26 & 29.2 & 404 & 46.2 \\
\hline Female & 578 & 55.6 & 78 & 70.8 & 500 & 53.8 \\
\hline \multicolumn{7}{|l|}{ Self-reported Race / Ethnicity } \\
\hline White, Non-Hispanic & 795 & 84.3 & 82 & 86.3 & 713 & 84.1 \\
\hline Black, Non-Hispanic & 138 & 8.8 & 17 & 8.0 & 121 & 8.9 \\
\hline Other & 75 & 6.9 & 5 & 5.6 & 70 & 7.0 \\
\hline \multicolumn{7}{|l|}{ Education $\dagger$} \\
\hline Less than High School & 218 & 17.4 & 31 & 23.2 & 187 & 16.7 \\
\hline High School / Equivalent & 386 & 37.8 & 45 & 46.0 & 341 & 36.8 \\
\hline Some College & 205 & 22.5 & 17 & 18.3 & 188 & 23.0 \\
\hline College Graduate & 199 & 22.3 & 11 & 12.5 & 188 & 23.5 \\
\hline \multicolumn{7}{|l|}{ Income } \\
\hline Less than $\$ 17,600$ & 256 & 22.5 & 41 & 32.7 & 215 & 21.3 \\
\hline$\$ 17,000-\$ 33,175$ & 249 & 21.5 & 34 & 30.8 & 215 & 20.5 \\
\hline$\$ 33,176-\$ 63,079$ & 259 & 25.7 & 15 & 15.0 & 244 & 27.0 \\
\hline$\$ 63,080+$ & 244 & 30.2 & 14 & 22.0 & 230 & 31.2 \\
\hline Currently Employed & 354 & 41.7 & 20 & 24.8 & 334 & 43.8 \\
\hline Health Limits Ability to Work & 289 & 25.8 & 71 & 68.8 & 218 & 20.8 \\
\hline \multicolumn{7}{|l|}{ Doctor Visits (In previous 2 y) $†$} \\
\hline 0-3 Doctor Visits & 294 & 30.6 & 25 & 22.1 & 269 & 31.6 \\
\hline 4-7 Doctor Visits & 270 & 26.4 & 17 & 18.9 & 253 & 27.3 \\
\hline 8-11 Doctor Visits & 181 & 17.5 & 22 & 24.9 & 159 & 16.7 \\
\hline 12 or More Doctor Visits & 263 & 25.4 & 40 & 34.1 & 223 & 24.4 \\
\hline Hospitalized (In previous 2 y) † & 260 & 23.0 & 36 & 34.0 & 224 & 21.8 \\
\hline Self-Report of No Medical Plan & 69 & 7.4 & 11 & 11.5 & 58 & 6.9 \\
\hline \multicolumn{7}{|l|}{$\mathrm{BMI}+$} \\
\hline$<25.0$ & 345 & 35.1 & 25 & 21.9 & 336 & 38.5 \\
\hline $25.0-29.9$ & 418 & 42.0 & 44 & 44.0 & 358 & 39.9 \\
\hline 30.0 or greater & 245 & 23.0 & 35 & 34.0 & 210 & 21.6 \\
\hline \multicolumn{7}{|l|}{ Smoking Status } \\
\hline Never Smoker & 403 & 40.0 & 38 & 34.0 & 365 & 40.8 \\
\hline Former Smoker & 456 & 45.3 & 46 & 49.1 & 410 & 44.8 \\
\hline Current Smoker & 149 & 14.7 & 20 & 16.9 & 129 & 14.4 \\
\hline \multicolumn{7}{|l|}{ Alcohol Consumption } \\
\hline Non-drinker & 525 & 48.6 & 77 & 70.5 & 448 & 46.0 \\
\hline Current Consumption & 483 & 51.4 & 27 & 29.6 & 456 & 54.0 \\
\hline Vigorous Activity (3 times per week) $\dagger$ & 427 & 44.4 & 34 & 34.5 & 393 & 45.6 \\
\hline \multicolumn{7}{|l|}{ Falls (In previous $2 \mathrm{y})$} \\
\hline None & 824 & 83.9 & 74 & 74.6 & 750 & 85.1 \\
\hline
\end{tabular}


Table 1 Characteristics of 2002 Health and Retirement Study restless legs syndrome module $(\mathbf{N}=\mathbf{1 , 0 0 8 )}($ Continued)

\begin{tabular}{|c|c|c|c|c|c|c|}
\hline Once & 103 & 9.3 & 12 & 10.3 & 91 & 9.1 \\
\hline 2 or More Times & 81 & 6.8 & 18 & 15.1 & 63 & 5.8 \\
\hline \multicolumn{7}{|l|}{ Self-Reported Health $\ddagger$} \\
\hline Very Good / Excellent & 417 & 43.8 & 17 & 16.0 & 400 & 47.1 \\
\hline Good & 342 & 35.3 & 28 & 34.3 & 314 & 35.4 \\
\hline Fair / Poor & 249 & 20.9 & 59 & 49.6 & 190 & 17.5 \\
\hline \multicolumn{7}{|l|}{ Self-Reported Pain $\$$} \\
\hline None & 733 & 73.9 & 28 & 29.8 & 705 & 79.1 \\
\hline Mild-Moderate & 111 & 10.4 & 20 & 16.9 & 91 & 9.6 \\
\hline Severe & 164 & 15.7 & 56 & 53.3 & 108 & 11.3 \\
\hline \multicolumn{7}{|l|}{ Self-Reported Medical Conditions } \\
\hline Hypertension $†$ & 520 & 48.9 & 66 & 60.5 & 454 & 47.5 \\
\hline Diabetes & 164 & 15.7 & 32 & 31.9 & 132 & 13.8 \\
\hline Cancer & 134 & 12.7 & 14 & 10.6 & 120 & 13.0 \\
\hline Lung Disease & 82 & 7.3 & 21 & 17.6 & 61 & 6.1 \\
\hline Heart Disease : & 241 & 21.6 & 40 & 38.2 & 201 & 19.6 \\
\hline Stroke & 63 & 5.2 & 8 & 6.9 & 55 & 5.0 \\
\hline Psychiatric Disease $\$$ & 128 & 13.2 & 33 & 34.1 & 95 & 10.7 \\
\hline Arthritis $\div$ & 592 & 54.5 & 87 & 83.1 & 505 & 51.1 \\
\hline \multicolumn{7}{|l|}{ Self-Reported Chronic Medications $\$$} \\
\hline None & 314 & 33.8 & 15 & 15.0 & 299 & 36.1 \\
\hline One Chronic Medication & 330 & 33.5 & 18 & 20.4 & 312 & 35.0 \\
\hline Two Chronic Medications & 214 & 19.0 & 26 & 22.2 & 188 & 18.6 \\
\hline Three or More Chronic Medications & 150 & 13.7 & 45 & 42.4 & 105 & 10.3 \\
\hline Depressive Symptoms (CES-D $\geq 2) \$$ & 219 & 19.8 & 57 & 46.6 & 162 & 16.6 \\
\hline ADL Limitation ( $\geq 1$ impairment) $\$$ & 136 & 12.4 & 40 & 39.2 & 96 & 9.2 \\
\hline IADL Limitation ( $\geq 1$ impairment) $*$ & 97 & 8.6 & 34 & 30.6 & 63 & 6.0 \\
\hline Mobility Limitation ( $\geq 1$ impairment) $\$$ & 454 & 41.3 & 91 & 87.6 & 363 & 35.8 \\
\hline Large Muscle Limitation ( $\geq 1$ impairment) $\ddagger$ & 611 & 56.7 & 98 & 95.1 & 513 & 52.2 \\
\hline Gross Motor Limitation ( $\geq 1$ impairment) & 222 & 20.1 & 61 & 56.1 & 161 & 15.8 \\
\hline Fine Motor Limitation ( $\geq 1$ impairment) & 112 & 10.1 & 26 & 28.7 & 86 & 7.9 \\
\hline
\end{tabular}

Abbreviations: $\mathrm{y}=$ Years; $\mathrm{ADL}=$ Activities of Daily Living; IADL = Instrumental Activities of Daily Living; BMI=Body Mass Index; CES-D=Center for Epidemiologic Studies Depression Scale.

* Percentage based on sampling weight to reflect U.S. population aged 54 or older at baseline, reflected in the underlying Health and Retirement Study cohort.

$\dagger \mathrm{x}^{2}$ test for association in all nominal variables and test for trend in all ordinal variables, $\mathrm{p}<0.05 ; \mathrm{p}<0.001$.

cessation of current medication (3\%), or other medication, not-further specified (27\%).

\section{Cross-sectional risk factors for RLS}

Multivariate regression modeling for potential RLS correlates was performed as described, using forward selection based on AIC improvements. Table 3 shows the full multivariate-adjusted model for factors cross-sectionally associated with RLS. Initially screened predictors of RLS that were not significant in multivariate modeling included race, education, marital status, geographic region, vigorous activity, smoking status, history of hospitalizations, depressive symptoms, or self-reported history of comorbidities (results not shown). The PR for gender was attenuated in the multivariate model. The older age groups were less likely to have RLS. Current alcohol consumption was associated with decreased prevalence of RLS, and modeling alcohol consumption based on frequency and/or amount per session did not improve the model (results not shown). Individuals with other sleep complaints (waking frequently at night or waking early and being unable to return to sleep) were more likely to also have RLS symptoms. Those more affected by bodily pain had significantly higher PR for 
Table 2 Prevalence* of symptoms related to restless leg syndrome in the 2002 Health and Retirement Study $(\mathrm{N}=\mathbf{1 , 0 0 8 )}$

\begin{tabular}{|c|c|c|c|c|c|c|}
\hline \multirow[t]{2}{*}{ Question } & \multirow[b]{2}{*}{$\begin{array}{l}\text { Total } \\
(\%)\end{array}$} & \multicolumn{3}{|c|}{ Age Group } & \multicolumn{2}{|c|}{ Gender } \\
\hline & & $\begin{array}{l}50-64 \\
(\%)\end{array}$ & $\begin{array}{l}65-79 \\
(\%)\end{array}$ & $\begin{array}{l}80+ \\
(\%)\end{array}$ & $\begin{array}{l}\text { Female } \\
(\%)\end{array}$ & $\begin{array}{l}\text { Male } \\
\text { (\%) }\end{array}$ \\
\hline Reported Leg Symptoms at Night & & & & & + & $t$ \\
\hline Never & 59.8 & 61.3 & 57.4 & 62.3 & 55.1 & 65.8 \\
\hline Less than once/month & 14.0 & 12.1 & 16.3 & 13.7 & 15.0 & 12.7 \\
\hline At least once/month but $<$ weekly & 6.3 & 7.4 & 5.7 & 4.1 & 6.6 & 5.9 \\
\hline At least once/week & 19.9 & 19.2 & 20.6 & 20.0 & 23.3 & 15.6 \\
\hline Crawling or aching arms or legs at rest & 37.9 & 39.8 & 36.4 & 35.7 & $44.0 \%$ & $30.2 \%$ \\
\hline Restless, fidgety, or unable sit still at rest & 25.2 & 28.1 & 23.5 & 20.2 & $27.9+$ & $21.7+$ \\
\hline Need to move, rub, or stretch legs at rest & 53.0 & 53.7 & 52.0 & 53.4 & $57.0 \%$ & $47.9 \%$ \\
\hline Twitching or kicking of arms or legs & 17.9 & $21.2+$ & $14.9+$ & $15.2+$ & $15.1+$ & $21.4+$ \\
\hline Itching sensations on body at rest & 20.9 & 20.2 & 21.2 & 22.5 & $25.2+$ & $15.6+$ \\
\hline Discussed problems with doctor $\S$ & 43.4 & $38.5+$ & $51.1+$ & $39.3+$ & 44.0 & 42.7 \\
\hline Prescribed treatment for restlessness \| & 40.4 & 33.3 & 43.0 & 54.1 & $47.8 \%$ & $29.8 *$ \\
\hline Treatment helped $\uparrow$ & 78.8 & 81.4 & 74.9 & 85.0 & 80.7 & 73.9 \\
\hline Case Definition ** & 10.6 & 11.9 & 9.7 & 8.4 & $13.5 \$$ & $7.0 \%$ \\
\hline
\end{tabular}

* = Prevalence estimates weighted to represent non-institutionalized U.S. population aged 54 or older.

$\mathrm{X}^{2}$ test for association in all dichotomous questions. Test for trend performed for RLS symptoms at night by gender. For age associations, test for trend was not-significant but $x^{2}$ test for nominal association was significant.

$+\mathrm{p}<0.05$.

$+\mathrm{p}<0.001$.

$\S$ Among those with any symptoms $(n=509)$.

$\|=$ Among those who sought medical advice $(n=239)$;

$\uparrow=$ Among those with prescribed treatment $(n=109)$.

** = Restless Legs symptoms defined as unpleasant or restless sensations in the legs at least once per month, and reporting "restless, fidgety, or unable to sit still" feelings at rest and the need to "move, rub, or stretch legs" $(n=104)$.

RLS, as did individuals whose health limits their ability to work, those with frequent falls, those reporting the highest levels of out-of-pocket medical expenses, and those reporting multiple medications for chronic conditions. However, those with more frequent visits to providers had lower PR for RLS after multivariate adjustment. Modeling BMI, total income, and medical expenses as continuous or quadratic functions did not improve the fit (results not shown). There was no evidence of meaningful effect modification in the predictors of RLS.

\section{RLS and concurrent disability}

Individuals with RLS were more likely to have certain functional status limitations at baseline (Table 4, adjusted for correlates reported in Table 3; specifically age, gender, moderate/severe pain, drinking status, BMI, health limiting ability to work, previous falls, other sleep symptoms, income, out-of-pocket medical expenses, frequency of provider visits, and number of chronic medications). RLS was not associated with significant PR ( $>0.20$, not shown) for several individual outcomes, including: difficulties walking short distances (across the room, one block, or requiring assistance to walk); difficulty climbing one flight of stairs; difficulties picking up a dime or carrying $10 \mathrm{lb}$; certain specific ADL limitations (feeding oneself); and most IADL limitations (reading a map, managing money, managing medications, or shopping for groceries). RLS sufferers have elevated PR for several aggregate functional outcomes (i.e., mobility limitations, large muscle group limitations, ADL, and IADL) but not aggregate gross motor and fine motor variables (Table 4).

\section{RLS and incident disability}

Next, we examined hazard ratios (HR) for incident functional limitations between RLS sufferers and nonsufferers for each disability outcome variable during the following three survey waves. Crude associations between RLS and incident disability ranged from roughly two- to four-fold increased rates (Table 5). However, with adjustment for baseline covariates (similar to the crosssectional analysis from Table 3, although allowing for time-dependent changes during follow-up), RLS did not predict significant increases for incident limitations of the aggregate measures (i.e, mobility limitations, ADL, IADL, gross and fine motor limitations, or large muscle 
Table 3 Multivariate-adjusted prevalence ratios for factors associated with restless legs syndrome* in the Health and Retirement Study 2002 survey $(\mathrm{N}=1008)$

\begin{tabular}{|c|c|c|c|}
\hline \multirow[t]{2}{*}{ Restless Leg Symptoms Predictor } & \multicolumn{3}{|c|}{ Multivariate Adjusted Model +} \\
\hline & PR & $95 \% \mathrm{Cl}$ & $\mathrm{p}$ \\
\hline \multicolumn{4}{|l|}{ Age (reference: $50-64$ years) } \\
\hline Age $65-79$ years & 0.45 & $(0.28,0.72)$ & $<0.001$ \\
\hline Age 80 or older & 0.52 & $(0.25,1.07)$ & 0.07 \\
\hline Gender (reference: Male) & 1.50 & $(0.93,2.40)$ & 0.10 \\
\hline \multicolumn{4}{|l|}{ Income (reference: Lowest Quartile) } \\
\hline 2nd quartile & 1.37 & $(0.83,2.27)$ & 0.22 \\
\hline 3rd quartile & 0.82 & $(0.46,1.46)$ & 0.51 \\
\hline 4th quartile & 1.54 & $(0.85,2.81)$ & 0.16 \\
\hline Current drinking status (reference: None) & 0.59 & $(0.37,0.92)$ & 0.02 \\
\hline \multicolumn{4}{|l|}{ Body Mass Index (reference: $\mathrm{BMI}<25.0$ ) } \\
\hline BMI $25.0-29.9$ & 1.77 & $(1.05,2.99)$ & 0.03 \\
\hline BMI 30 or greater & 1.40 & $(0.80,2.46)$ & 0.24 \\
\hline \multicolumn{4}{|l|}{ Pain category (reference: None) } \\
\hline Mild to moderate & 2.67 & $(1.47,4.84)$ & 0.001 \\
\hline Severe & 3.44 & $(2.00,5.93)$ & $<0.001$ \\
\hline \multicolumn{4}{|c|}{ Number of chronic medications used (reference: None) } \\
\hline 1 chronic medication & 1.14 & $(0.57,2.26)$ & 0.71 \\
\hline 2 chronic medications & 1.32 & $(0.70,2.46)$ & 0.39 \\
\hline 3 or more chronic medications & 2.54 & $(1.26,5.12)$ & 0.009 \\
\hline \multicolumn{4}{|c|}{ Out of pocket medical expenses (reference: Highest Quartile) } \\
\hline 2nd quartile & 1.65 & $(0.88,3.09)$ & 0.12 \\
\hline 3rd quartile & 1.33 & $(0.69,2.57)$ & 0.40 \\
\hline 4th quartile & 2.12 & $(1.17,3.86)$ & 0.01 \\
\hline \multicolumn{4}{|c|}{ Frequency of doctor visits in last 2 years (reference: $0-3$ visits) } \\
\hline 4-7 visits & 0.89 & $(0.43,1.82)$ & 0.74 \\
\hline 8-11 visits & 0.82 & $(0.46,1.46)$ & 0.50 \\
\hline 12 or more visits & 0.49 & $(0.27,0.90)$ & 0.02 \\
\hline Health limits ability to work & 2.91 & $(1.75,4.85)$ & $<0.001$ \\
\hline \multicolumn{4}{|l|}{ Falls in the last 2 years (reference: None) } \\
\hline Fell once & 1.33 & $(0.73,2.40)$ & 0.35 \\
\hline Fell 2 or more times & 2.63 & $(1.49,4.66)$ & $<0.001$ \\
\hline \multicolumn{4}{|l|}{ Associated sleep problems } \\
\hline Problems with early waking & 1.69 & $(1.09,2.62)$ & 0.02 \\
\hline Problems with frequent waking & 1.55 & $(1.00,2.41)$ & 0.05 \\
\hline
\end{tabular}

Abbreviations: $\mathrm{PR}=$ prevalence ratio; $\mathrm{Cl}=$ confidence interval; $\mathrm{BMI}=$ Body Mass Index.

* Restless Legs symptoms defined as unpleasant or restless sensations in the legs at least once per week, and reporting "restless, fidgety, or unable to sit still" feelings at rest and the need to "move, rub, or stretch legs" $(n=104)$.

† Final multivariate model reflecting automated variable forward selection with improvements to Akaike Information Criterion statistic.

groups), although mobility limitations approached statistical and clinical significance (HR: 1.90, 95\% confidence interval $(0.97,3.70), \mathrm{p}=0.06)$. RLS did predict increases in some specific functions, including difficulties: climbing several stair flights; raising from a chair; sitting prolonged periods; stooping or kneeling; pushing or pulling large objects; picking up a dime; lifting $10 \mathrm{lb}$; or reaching arms up). Again, no limitation was seen for walking short distances. No specific ADL or IADL functions were significantly different for RLS sufferers during follow-up. 
Table 4 Cross-sectional associations (Prevalence Ratios) between restless legs syndrome* and functional limitations in the Health and Retirement Study 2002 survey $(\mathrm{N}=1008)$

\begin{tabular}{|c|c|c|c|c|c|c|}
\hline Functional Limitation Outcome & Total (N) & Limited (N) & $\%$ & $\mathrm{PR}^{*}$ & $95 \% \mathrm{Cl}$ & $\mathbf{P}$ \\
\hline Mobility limitations (At least 1 ) & 1008 & 454 & 45.0 & 1.25 & $(1.08,1.46)$ & 0.004 \\
\hline Some difficulty walking several blocks & 994 & 273 & 27.5 & 1.43 & $(1.12,1.82)$ & 0.004 \\
\hline Difficulty climbing several flights & 900 & 408 & 45.3 & 1.16 & $(0.98,1.36)$ & 0.09 \\
\hline Large muscle group limitations (At least 1 ) & 1008 & 611 & 60.6 & 1.20 & $(1.06,1.36)$ & 0.004 \\
\hline Some difficulty sitting for 2 hours & 997 & 191 & 19.2 & 1.29 & $(0.95,1.76)$ & 0.11 \\
\hline Some difficulty rising from chair & 1007 & 397 & 39.4 & 1.20 & $(0.96,1.49)$ & 0.11 \\
\hline Difficulty with stooping & 997 & 451 & 45.2 & 1.15 & $(0.96,1.38)$ & 0.14 \\
\hline Pushing or pulling a large object & 957 & 227 & 23.7 & 1.56 & $(1.18,2.07)$ & 0.002 \\
\hline Gross motor limitationst (At least 1 ) & 1008 & 222 & 22.0 & 1.13 & $(0.86,1.49)$ & 0.37 \\
\hline Fine motor limitations & 1008 & 112 & 11.1 & 1.42 & $(0.89,2.26)$ & 0.14 \\
\hline Difficulty reaching or extending arms up & 1007 & 139 & 13.8 & 1.68 & $(1.10,2.55)$ & 0.02 \\
\hline ADL limitations (At least 1 ) & 1008 & 136 & 13.5 & 1.46 & $(1.00,2.11)$ & 0.05 \\
\hline \multicolumn{7}{|l|}{ Specific ADLs } \\
\hline Dressing oneself & 1008 & 74 & 7.3 & 2.23 & $(1.26,3.95)$ & 0.006 \\
\hline Bathing oneself & 1008 & 53 & 5.3 & 1.69 & $(0.80,3.59)$ & 0.17 \\
\hline Transferring into or out of bed & 1007 & 46 & 4.6 & 2.07 & $(1.01,4.23)$ & 0.05 \\
\hline Toileting & 1008 & 51 & 5.1 & 2.16 & $(1.04,4.52)$ & 0.04 \\
\hline IADL limitations (At least 1) & 1008 & 97 & 9.6 & 1.71 & $(1.13,2.59)$ & 0.01 \\
\hline \multicolumn{7}{|l|}{ Specific IADLs } \\
\hline Using a telephone & 1008 & 20 & 2.0 & 0.26 & $(0.06,1.22)$ & 0.09 \\
\hline Preparing Meals & 952 & 43 & 4.5 & 2.96 & $(1.47,5.95)$ & 0.002 \\
\hline
\end{tabular}

* Restless Legs symptoms defined as unpleasant or restless sensations in the legs at least once per week, and reporting "restless, fidgety, or unable to sit still" feelings at rest and the need to "move, rub, or stretch legs" $(n=104)$. Adjusted for significant covariates from analysis in Table 3, including: age, gender, income category (quartiles), health interfering with ability to work, pain symptoms (mild-moderate or severe compared to none), other sleep symptoms (walking too easily or too early), body mass index, current drinking status, number of chronic medications used, out-of-pocket medical expenses (quartiles), number of doctor visits in the last $2 \mathrm{y}$, and falls in the previous $2 \mathrm{y}$.

+ Gross motor limitations defined as at least some difficulty in one of these domains: Walking 1 block, walking with an assistive device, climbing 1 flight of stairs, transferring, or bathing.

+ Fine motor limitations defined as at least some difficulty in one of these domains: Picking up a dime, feeding oneself, or dressing oneself.

Abbreviations: $\mathrm{PR}=$ prevalence ratio; $\mathrm{Cl}=$ confidence interval; $\mathrm{ADL}=$ Activities of Daily Living; $\mathrm{IADL}=$ Instrumental Activities of Daily Living; $\mathrm{y}=$ years.

\section{Discussion}

This population-based sample of U.S. elders confirms that restless legs syndrome is common, with $10.6 \%$ of our sample meeting the operational definition of weekly RLS symptoms, and potentially disabling. The prevalence estimates are consistent with other reports, although we were unable to classify patients with primary versus secondary RLS. The multinational RLS Epidemiology, Symptoms, and Treatment (REST) study found annual period prevalence of $7.2 \%$, although $5 \%$ had weekly symptoms [5]. The 2005 National Sleep Foundation Poll's estimate was $9.7 \%$ for symptoms several nights per week [4]. The MEMO study, another population-based survey, reported $9.8 \%$ prevalence in elders in Germany [7]. Secondary RLS can be very common, such as reports of $20 \%$ in hemodialysis patients [10], although primary RLS is less common, estimated at $2.4 \%$ in the U.S. [9].
Among those reporting RLS symptoms, fewer than half sought medical care. Among those who discuss their symptoms, fewer than half received treatment. However, most who received treatment reported symptom improvement. This pattern confirms reports from the REST study, suggesting delays in both diagnosis of RLS and initiation of appropriate therapies [5]. The literature suggests RLS patients have good response to therapy in $90 \%$ of patients when correctly diagnosed [2]. Evidencebased guidelines for RLS treatment now exist [23], although one clinic-based German study found no clinical improvement with evidence-based guideline adherence [24]. In the REST study, 81\% of RLS patients with distressing symptoms discussed their symptoms with their physicians, but only $24 \%$ of these were given any diagnosis $[3,5]$. The majority of patients with RLS were diagnosed with circulation problems, arthritis, back or spine problems, varicose veins, depression, anxiety, or trapped 
Table 5 Incident functional limitations predicted by Restless Legs Syndrome* at baseline $(2002, N=1008)$ in the Health and Retirement Study (Follow-up until 2008)

\begin{tabular}{|c|c|c|c|c|c|c|c|c|}
\hline \multirow[t]{2}{*}{ Functional Limitation Outcome } & \multirow[b]{2}{*}{ At Risk } & \multirow[b]{2}{*}{ Disability } & \multicolumn{3}{|c|}{ Unadjusted $\dagger$} & \multicolumn{3}{|c|}{ Multivariate Adjusted $\dagger$} \\
\hline & & & HR & $95 \% \mathrm{Cl}$ & $\mathrm{p}$ & HR & $95 \% \mathrm{Cl}$ & $p$ \\
\hline Mobility limitations & 524 & 247 & 2.26 & $(1.21,4.23)$ & 0.01 & 1.90 & $(0.97,3.70)$ & 0.06 \\
\hline Difficulty climbing several flights & 564 & 246 & 3.13 & $(2.03,4.85)$ & $<0.001$ & 2.38 & $(1.39,4.06)$ & 0.002 \\
\hline Difficulty climbing one flight & 780 & 180 & 3.62 & $(2.36,5.55)$ & $<0.001$ & 1.51 & $(0.96,2.40)$ & 0.08 \\
\hline Large muscle group limitations & 371 & 210 & 1.97 & $(0.86,4.53)$ & 0.11 & 1.01 & $(0.48,2.12)$ & 0.98 \\
\hline Some difficulty sitting for 2 hours & 745 & 178 & 3.26 & $(2.12,5.01)$ & $<0.001$ & 2.17 & $(1.25,3.75)$ & 0.006 \\
\hline Some difficulty rising from chair & 565 & 224 & 3.16 & $(2.11,4.73)$ & $<0.001$ & 2.54 & $(1.62,3.99)$ & $<0.001$ \\
\hline Difficulty with stooping & 518 & 245 & 4.09 & $(2.86,5.86)$ & $<0.001$ & 2.66 & $(1.71,4.15)$ & $<0.001$ \\
\hline Pushing or pulling a large object & 721 & 238 & 3.23 & $(2.01,5.19)$ & $<0.001$ & 1.79 & $(1.08,2.99)$ & 0.02 \\
\hline Gross motor limitationst (At least 1 ) & 731 & 212 & 2.43 & $(1.46,4.03)$ & 0.001 & 0.91 & $(0.52,1.59)$ & 0.73 \\
\hline Fine motor limitations: (At least 1 ) & 826 & 154 & 2.45 & $(1.50,4.01)$ & $<0.001$ & 1.18 & $(0.72,1.94)$ & 0.52 \\
\hline Difficulty with lifting or carrying $10 \mathrm{lb}$ & 744 & 206 & 3.02 & $(1.99,4.59)$ & $<0.001$ & 1.61 & $(1.05,2.46)$ & 0.03 \\
\hline Difficulty picking up a dime & 872 & 89 & 2.97 & $(1.69,5.21)$ & $<0.001$ & 1.97 & $(1.12,3.46)$ & 0.02 \\
\hline Difficulty reaching or extending arms up & 799 & 173 & 3.22 & $(2.06,5.06)$ & $<0.001$ & 1.76 & $(1.05,2.97)$ & 0.03 \\
\hline ADL limitations (At least 1 ) & 807 & 162 & 1.94 & $(1.11,3.40)$ & 0.02 & 0.64 & $(0.36,1.13)$ & 0.12 \\
\hline IADL limitations (At least 1 ) & 843 & 139 & 2.62 & $(1.50,4.57)$ & 0.001 & 1.05 & $(0.60,1.82)$ & 0.87 \\
\hline
\end{tabular}

* Restless Legs symptoms defined as unpleasant or restless sensations in the legs at least once per week, and reporting "restless, fidgety, or unable to sit still" feelings at rest and the need to "move, rub, or stretch legs" ( $n=104$ in 2002).

† Unadjusted hazard ratio shows incident disability. Functional limitation was assessed biannually, subjects censored if no longer eligible at follow-up waves. Multivariate-adjusted hazard ratio includes variables adjusted for in Table 3 (cross-sectionally associated with RLS, including: age, gender, income category (quartiles), health interfering with ability to work, pain symptoms (mild-moderate or severe compared to none), other sleep symptoms (walking too easily or too early), body mass index, current drinking status, number of chronic medications used, out-of-pocket medical expenses (quartiles), number of doctor visits in the last $2 \mathrm{y}$, and falls in the previous $2 \mathrm{y}$ ), as well as time-varying covariates associated with RLS in the follow-up waves (incident stroke, incident psychiatric diagnosis).

† Gross motor limitations defined as at least some difficulty in one of these domains: Walking 1 block, walking with an assistive device, climbing 1 flight of stairs, transferring, or bathing.

+ Fine motor limitations defined as at least some difficulty in one of these domains: Picking up a dime, feeding oneself, or dressing oneself.

Abbreviations: $\mathrm{HR}=$ hazard ratio; $\mathrm{Cl}=$ confidence interval; $\mathrm{ADL}=$ Activities of Daily Living; $\mathrm{IADL}=$ Instrumental Activities of Daily Living; $y=$ years; $\mathrm{lb}=$ pounds.

nerves, but only $6 \%$ were correctly diagnosed with RLS [5].

This study identifies associations between RLS and disability using valid functional limitation measures. RLS sufferers were $46 \%$ more likely to have ADL limitations at baseline, adjusted for covariates, and several ADL domains had doubled risk (dressing oneself, transferring, and toileting). RLS sufferers were also $71 \%$ more likely to have IADL limitations, including nearly three-fold increase in difficulty making meals by themselves. RLS sufferers were more likely to have concurrent mobility limitations (25\% increase) and limitations requiring large muscle groups (20\% increase), but did not have significant changes in fine or gross motor functions. However, some specific limitations, such as lifting their arms, pushing heavy weights, and rising from a chair, were significantly increased. Associations with mobility measures were more prominent in more strenuous tasks (multiple blocks or multiple stair flights).

This study is the first population-based study to longitudinally assess functional measures among adults with RLS. Despite adjustment for all baseline and time- dependent covariates, specific functional limitations were significantly increased among subjects with RLS. RLS suffers were more likely to develop specific incident disabilities, which involving the core, lower extremities, and upper extremities, such as difficulty climbing stairs $(\mathrm{HR}=$ $2.38)$, difficulty stooping $(\mathrm{HR}=2.66)$, difficulty rising from a chair $(H R=2.54)$, difficulty sitting for 2 hours $(\mathrm{HR}=2.17)$, moving large objects $(\mathrm{HR}=1.79)$, raising arms $(H R=1.76)$, or picking up a dime $(H R=1.97)$. However, no aggregate measures (ADL, IADL, gross motor, fine motor, mobility, or large muscle group limitations) reached statistical significance during follow-up, after adjustment for baseline characteristics (particularly pain and chronic medication use). If pain mediates how RLS affects disability, this may reflect over-adjustment. Also, excluding disabled patients at baseline markedly decreased the population at-risk for new disability, limiting the power of the study. We did not model transitions from mild to more severe disability.

The difficulty with prolonged sitting follows with the expected perturbation of RLS symptoms during periods of inactivity. Sleep symptoms (frequent waking or waking 
early without being able to fall asleep) were significantly associated with RLS. We adjusted for these sleep symptoms when analyzing the potential impact of RLS on disability, although symptoms interfering with sleep quality may be an intermediate factors between RLS and disability. Multiple studies have considered role limitations and quality of life. RLS patients have been shown to have decreased scores on the Medical Outcomes Study 36Item Short Form (SF-36) in all tested domains $[5,15]$. The National Sleep Foundation (NSF) poll noted that RLS sufferers were more likely to be unemployed and to have difficulties at work [4]. We confirmed that employed elders outside of the home were less likely to have RLS (age- and sex- adjusted PR 0.38, 95\% CI 0.210.68 , although not significant in multivariate analysis), and those who report that there health limited their ability to work were three times more likely to have RLS. The REST study reported that RLS sufferers reported daytime sleepiness (32\%), difficulty concentrating (15\%), and negative influences on daily activities and personal and work life [5]. The NSF poll also found evidence of daytime symptoms in patients with RLS [4]. These findings were replicated in a geriatric sample, with poor sleep quality, daytime somnolence, and low social functioning due to impaired tolerance for inactivity [16]. One Scandinavian study showed similar impact on sleep quality, but noted lower SF-12 physical domain scores but not mental domain scores [6]. An analysis of health-related quality of life (HRQOL) in RLS patients in multiple sites in Germany suggested that sleep deficits, duration of symptoms, and household income mediated RLS associates with clinical rating scales, with RLS effects consistent with other chronic neurologic diseases [13]. A Swedish study, limited to women, suggested statistically and clinically significant deviations in HRQOL although mental domains predominated [14]. When HRQOL was assessed in the REST study, McKrink et al. were able to estimate factors related to RLS diagnosis, severity, and treatment using SF-36 as predictors of the domains, but no comparison to patients without RLS was possible [12]. One recent small study of RLS patients showed substantial cognitive deficits similar to those deprived of sleep for one night, despite assessment at a time when RLS symptoms were not active [25]. We did not find concomitant cognitive dysfunction associated with RLS (results not shown).

Our risk factor analysis yielded results consistent with other studies. Women were nearly twice as likely to have RLS based on age-adjusted estimates; however, this difference was reduced by half when adjusted for other covariates, such as pain syndromes, alcohol consumption, BMI, age, medication use, and health care utilization, suggesting that gender differences in RLS prevalence may be partially due to confounding. There was no evidence of significant effect modification by gender, although there was a relatively small number of males with RLS $(n=26)$. Although RLS is thought to be more common in the elderly, the prevalence of RLS trended downward with age in our sample. Several studies report increased prevalence with age, peaking around age $65[4,5]$, consistent with our reference group, which was those aged 50-64.

RLS is known to have a strong hereditary component [8] but may be associated with acquired factors such as increased BMI, inactivity, and low alcohol consumption [26]. Vigorous activity was crudely associated with RLS but the association did not persist in multivariate modeling. We observed that those with some alcohol consumption tended to have decreased prevalence of RLS, similar to Phillips and colleagues [26], despite modeling alcohol consumption in various ways. There were no independent effects for "binge drinking", frequency of drinking, or amount per session. Cigarette smoking associations have been inconsistent $[4,6,7,26,27]$ and smoking status was not associated with RLS in this sample. Crude associations with arthritis, hypertension, diabetes, lung disease, and heart disease were observed, but adjustment for general health status and symptomatic variables were better predictors of RLS cross-sectionally. Although RLS may be secondary to conditions like COPD [6], anemia, kidney disease, and diabetes, we did not observe persistent effects for medical comorbidities in this study. Psychiatric comorbidity or depressive symptoms also did not predict RLS after adjustment, despite reports that mood disorders are more common in RLS sufferers [7,28-30]. We did adjust for medication use and sleep-related symptoms, which may obscure this association. Similarity, self-reported health status had previously been noted as a predictor of RLS [26], but did not retain significance in our study. RLS is common in patients with fibromyalgia [31,32], and sleep quality can be a determinant of HRQOL in these patients. Heavy medication use was associated with RLS, although we could not assess if this was causal or merely an indicator of multiple comorbidities.

Pain experience was a strong predictor of RLS, as was history of frequent falls. There may be overlap between RLS and chronic neuropathic pain pathophysiology, as both are mediated by the dopaminergic system [33]. Neuropathic pain treatments are effective on RLS symptoms [33], and non-opioid analgesic use predicted RLS among patients taking tricyclic and serotonin-reuptakeinhibiting antidepressants [34]. Most RLS sufferers in that study had chronic recurrent pain, and nearly all used analgesics [35]. RLS patients are more sensitive to pain, measured by pinprick hyperalgesia in the extremities [36]. Furthermore, RLS patients showed worse bodily pain scores using SF-36 [15]. Pain is not recognized 
in the standardized definition of RLS, but if pain is part of the clinical syndrome, increased pain may, at least partially, explain RLS associations with incident functional limitations. Our findings support the hypothesis that RLS sufferers may suffer from other chronic pain syndromes. Or, patients with neuropathic pain syndromes may some symptoms that mimic RLS [9].

There are limitations to this work. First, the 2002 RLS module was part of the pre-existing HRS cohort and not a principle aim for the study, and data on RLS was collected at one time point and was without clinical evaluation. Risk factors and correlates, including functional limitations, were available both before and after this assessment. We did not differentiate between primary and secondary RLS. Standardized diagnostic criteria suggested by the International RLS Study Group [2] were not strictly applied, but our case definition included the essence of the core diagnostic criteria at least weekly. Our case definition did not require the characteristics of movement providing symptom alleviation or that the symptoms worsen at night, which are considered diagnostic criteria for RLS. However, the questions did specify that the symptoms were present at rest. Our prevalence was consistent with other studies in elders, but extrapolation of our findings should be limited to those aged 54 or older. Although unmeasured confounding is always a concern, we also risk over-adjustment in multivariate modeling. Some crude estimates were markedly changed with control for pain and medication use. We do not know if pain is a mediating variable or part of the clinical syndrome rather than a true confounder. We also did not confirm chronic diseases as covariates with RLS as seen in other studies, but our general markers of health status and medication use better fit the data. We also could not assess for specific medication class effects, such as medications that cause akathisia (i.e., anti-psychotics and anti-depressants) [37], which has clinical similarities to RLS. Some of the RLS treatments reported by subjects (e.g., "surgery or epidural") could be potentially disabling. Although we could detect incident functional limitations, we excluded subjects with specific disabilities at baseline. For the most common disabilities, this reduced the sample size considered at risk, which also decreased the power to detect differences. Furthermore, as RLS was only assessed at one time-point , associations that may be "early" or "late" manifestations of disability would be differentially detected.

\section{Conclusions}

In this study, we have corroborated reports that RLS may be substantially under-diagnosed and undertreated during this period. RLS predicted functional decline in multiple domains, which suggests an impact on subjects' abilities to work and live independently. If the observed limitations are reversible when appropriate treatment is given, early detection and treatment of RLS may help remediate RLS functional decline.

\section{Competing interests}

The authors declare that they have no competing interests.

\section{Authors' contributions}

DJC conducted the statistical analyses and drafted the manuscript. Both authors were involved in the conception of the research and interpretation of the data. RBW is a co-principle investigator for HRS, has ongoing involvement in the design and maintenance of the cohort, and was responsible for devising the survey questions use in this manuscript. Both authors read and approved of the final manuscript.

\section{Acknowledgements}

The authors acknowledge Dr. Joseph E. Cavanaugh from the University of lowa and Dr. Mary Vaughan Sarrazin of the lowa City Veterans Affairs' Center for Comprehensive Access \& Delivery Research and Evaluation for providing statistical advice for the analyses included in this manuscript. Both authors had full access to all of the data in the study and take responsibility for the integrity of the data and the accuracy of the data analysis. This work was supported by the National Institute on Aging (NIA Grant U01 AG00 9740). Dr. Cirillo was partially supported by the University of lowa Medical Scientist Training Program (NIGMS T32 GM07337). The funding agencies had no role in design or conduct of the study.

Received: 11 November 2011 Accepted: 12 July 2012

Published: 26 July 2012

\section{References}

1. Clark MM: Restless legs syndrome. J Am Board Fam Pract 2001, 14(5):368-374.

2. Allen RP, Picchietti D, Hening WA, Trenkwalder C, Walters AS, Montplaisi J: Restless legs syndrome: diagnostic criteria, special considerations, and epidemiology. A report from the restless legs syndrome diagnosis and epidemiology workshop at the National Institutes of Health. Sleep Med 2003, 4(2):101-119.

3. Hening W, Walters AS, Allen RP, Montplaisir J, Myers A, Ferini-Strambi L: Impact, diagnosis and treatment of restless legs syndrome (RLS) in a primary care population: the REST (RLS epidemiology, symptoms, and treatment) primary care study. Sleep Med 2004, 5(3):237-246.

4. Phillips B, Hening W, Britz P, Mannino D: Prevalence and correlates of restless legs syndrome: results from the 2005 National Sleep Foundation Poll. Chest 2006, 129(1):76-80.

5. Allen RP, Walters AS, Montplaisir J, Hening W, Myers A, Bell TJ, Ferini-Strambi L: Restless legs syndrome prevalence and impact: REST general population study. Arch Intern Med 2005, 165(11):1286-1292.

6. Benediktsdottir B, Janson C, Lindberg E, Arnardottir ES, Olafsson I, Cook E, Thorarinsdottir EH, Gislason T: Prevalence of restless legs syndrome among adults in Iceland and Sweden: Lung function, comorbidity, ferritin, biomarkers and quality of life. Sleep Med 2010, 11(10):1043-1048.

7. Rothdach AJ, Trenkwalder C, Haberstock J, Keil U, Berger K: Prevalence and risk factors of RLS in an elderly population: the MEMO study. Memory and Morbidity in Augsburg Elderly. Neurology 2000, 54(5):1064-1068.

8. Zucconi M, Ferini-Strambi L: Epidemiology and clinical findings of restless legs syndrome. Sleep Med 2004, 5(3):293-299.

9. Allen RP, Bharmal M, Calloway M: Prevalence and disease burden of primary restless legs syndrome: results of a general population survey in the United States. Mov Disord 2011, 26(1):114-120.

10. Salman SM: Restless legs syndrome in patients on hemodialysis. Saudi J Kidney Dis Transpl 2011, 22(2):368-372.

11. Walters AS, Hickey K, Maltzman J, Verrico T, Joseph D, Hening W, Wilson V, Chokroverty S: A questionnaire study of 138 patients with restless legs syndrome: The 'Night-Walkers' survey. Neurology 1996, 46(1):92-95.

12. McCrink L, Allen RP, Wolowacz S, Sherrill B, Connolly M, Kirsch J: Predictors of health-related quality of life in sufferers with restless legs syndrome: a multi-national study. Sleep Med 2007, 8(1):73-83.

13. Happe S, Reese JP, Stiasny-Kolster K, Peglau I, Mayer G, Klotsche J, Giani G, Geraedts M, Trenkwalder C, Dodel R: Assessing health-related quality of 
life in patients with restless legs syndrome. Sleep Med 2009, 10(3):295-305

14. Wesstrom J, Nilsson S, Sundstrom-Poromaa I, Ulfberg J: Health-related quality of life and restless legs syndrome among women in Sweden. Psychiatry Clin Neurosci 2010, 64(5):574-579.

15. Abetz L, Allen R, Follet A, Washburn T, Earley C, Kirsch J, Knight H: Evaluating the quality of life of patients with restless legs syndrome. Clin Ther 2004, 26(6):925-935.

16. Cuellar NG, Strumpf NE, Ratcliffe SJ: Symptoms of restless legs syndrome in older adults: outcomes on sleep quality, sleepiness, fatigue, depression, and quality of life. J Am Geriatr Soc 2007, 55(9):1387-1392.

17. An Overview of Health and Retirement Study Components. http://hrsonline.isr. umich.edu.

18. Overview of HRS Public Data Files for Cross-sectional and Longitudinal Analysis. http://hrsonline.isr.umich.edu/docs/dmgt/OverviewofHRSPublicData.pdf.

19. Steffick DE: Documentation of Affective Functioning Measures in the Health and Retirement Study. Ann Arbor, MI: University of Michigan; 2000.

20. Barros AJD, Hirakata VN: Alternatives for logistic regression in cross-sectional studies: an empirical comparison of models that directly estimate the prevalence ratio. BMC Med Res Methodol 2003, 3:21.

21. Kleinbaum DG, Kupper LL, Muller KE, Nizum A: Applied Regression Analysis and Other Multivariate Methods. 3rd edition. Detriot: Duxbury Press; 1998.

22. Greenland S: Modeling and variable selection in epidemiologic analysis. Am J Public Health 1989, 79(3):340-349.

23. Trenkwalder C, Hening WA, Montagna P, Oertel WH, Allen RP, Walters AS, Costa J, Stiasny-Kolster K, Sampaio C: Treatment of restless legs syndrome: an evidence-based review and implications for clinical practice. Mov Disord 2008, 23(16):2267-2302.

24. Godau J, Spinnler N, Wevers AK, Trenkwalder C, Berg D: Poor effect of guideline based treatment of restless legs syndrome in clinical practice. J Neurol Neurosurg Psychiatry 2010, 81(12):1390-1395.

25. Pearson VE, Allen RP, Dean T, Gamaldo CE, Lesage SR, Earley CJ: Cognitive deficits associated with restless legs syndrome (RLS). Sleep Med 2006, 7(1):25.

26. Phillips B, Young T, Finn L, Asher K, Hening WA, Purvis C: Epidemiology of restless legs symptoms in adults. Arch Intern Med 2000, 160(14):2137-2141.

27. Lavigne GL, Lobbezoo F, Rompre PH, Nielsen TA, Montplaisir J: Cigarette smoking as a risk factor or an exacerbating factor for restless legs syndrome and sleep bruxism. Sleep 1997, 20(4):290-293.

28. Hornyak M, Kopasz M, Berger M, Riemann D, Voderholzer U: Impact of sleep-related complaints on depressive symptoms in patients with restless legs syndrome. J Clin Psychiatry 2005, 66(9):1139-1145.

29. Sevim S, Dogu O, Kaleagasi H, Aral M, Metin O, Camdeviren H: Correlation of anxiety and depression symptoms in patients with restless legs syndrome: a population based survey. J Neurol Neurosurg Psychiatry 2004, 75(2):226-230

30. Winkelmann J, Prager M, Lieb R, Pfister H, Spiegel B, Wittchen HU, Holsboer F, Trenkwalder C, Strohle A: "Anxietas tibiarum". Depression and anxiety disorders in patients with restless legs syndrome. J Neurol 2005, 252(1):67-71.

31. Viola-Saltzman M, Watson NF, Bogart A, Goldberg J, Buchwald D: High prevalence of restless legs syndrome among patients with fibromyalgia: a controlled cross-sectional study. J Clin Sleep Med 2010, 6(5):423-427.

32. Yunus MB, Aldag JC: Restless legs syndrome and leg cramps in fibromyalgia syndrome: a controlled study. BMJ 1996, 312(7042):1339.

33. Stiasny-Kolster K, Trenkwalder C, Fogel W, Greulich W, Hahne M, Lachenmayer L, Oechsner M, Oertel WH: Restless legs syndrome--new insights into clinical characteristics, pathophysiology, and treatment options. J Neurol 2004, 251(Supp 6):VI39-VI43.

34. Leutgeb U, Martus P: Regular intake of non-opioid analgesics is associated with an increased risk of restless legs syndrome in patients maintained on antidepressants. Eur J Med Res 2002, 7(8):368-378.

35. Leutgeb U, Schmelz M, Koppert W: Altered central excitability and analgesic treatment in patients with restless legs syndrome. Brain 2005, 128(6):E34.

36. Stiasny-Kolster K, Magerl W, Oertel WH, Moller JC, Treede RD: Static mechanical hyperalgesia without dynamic tactile allodynia in patients with restless legs syndrome. Brain 2004, 127(4):773-782.

37. Gerber PE, Lynd LD: Selective serotonin-reuptake inhibitor-induced movement disorders. Ann Pharmacother 1998, 32(6):692-698. doi:10.1186/1471-2318-12-39

Cite this article as: Cirillo and Wallace: Restless legs syndrome and functional limitations among American elders in the Health and Retirement Study. BMC Geriatrics 2012 12:39.

\section{Submit your next manuscript to BioMed Central and take full advantage of:}

- Convenient online submission

- Thorough peer review

- No space constraints or color figure charges

- Immediate publication on acceptance

- Inclusion in PubMed, CAS, Scopus and Google Scholar

- Research which is freely available for redistribution 